Volume 9 No.2, March -April 2020

International Journal of Advanced Trends in Computer Science and Engineering

Available Online at http://www.warse.org/IJATCSE/static/pdf/file/ijatcse60922020.pdf

https://doi.org/10.30534/ijatcse/2020/60922020

\title{
Application of Matrices in Flipping the Image Using Python Program
}

\author{
Vinitha Navis Varuvel ${ }^{1}$, A.Kanchana ${ }^{2}$ \\ ${ }^{1}$ Assistant Professor, Department of Humanities and Science, Rajalakshmi Institute of Technology, Chennai, India, Email: \\ vinitha.navis@gmail.com \\ ${ }^{2}$ Assistant Professor, Department of Humanities and Science, Rajalakshmi Institute of Technology, Chennai, India, Email: \\ kanchana.anbazhagan@gmail.com
}

\begin{abstract}
Matrices have various applications, one of them being rotating or flipping a picture. A flipped image is required while printing images on a surface, designing circuits and taking selfies in a mobile phone. Otherwise, the original image will remain as a mirror image. Here, we have used a simple python program which corrects the mirror image with the help of matrix row operation and transpose of a matrix.
\end{abstract}

Key words: Matrix, Python programming language, Flipping of an image.

\section{INTRODUCTION}

A flipped image refers to an image that is generated by a mirror-reversal of the original image with respect to its horizontal axis, since mirrors reverse images in one dimension. A flip (mirror effect) is done by reversing the pixels horizontally. i.e. the pixel situated at co-ordinate $(x, y)$ will be situated at co-ordinate (width-x-1,y) in the new image. Here, the matrix [1]operations play a vital role.

While taking selfies, we notice that a lady wearing a saree from the right to left appears as if she is wearing it the other way, making the picture look awkward. Thus, a selfie appears the same way as a mirror image. The reason behind this is the difference between reflection and rotational symmetry. So, in reality, we have looked into its reflection and not the 180degree rotation. Hence, in order to bring the photo back to the original form, it must be rotated [2].

In order to get the printing of images on fabrics, glasses and other items, rotation or flipping of an image should be done to get the desired output. However, this should be done before the image gets printed. In addition, to design a circuit connection into a printed circuit boards, flipping must be applied to an image. The process of flipping an image with the help of matrix operations has wide applications in the field of industry, medicine and other technical applications.

\section{MATERIAL AND METHODS}

The following materials and methods are used in this paper:

\subsection{Matrix}

From reference [3], it can be noted that the transpose of a matrix flips a matrix over its diagonals. The transpose of a matrix, written as $A^{\mathrm{T}}$, is the matrix got by interchanging the rows and columns [4] and [5]. The $\mathrm{i}^{\text {th }}$ row and $\mathrm{j}^{\text {th }}$ column of $\mathrm{A}$ will be transformed to the $\mathrm{j}^{\text {th }}$ row and $\mathrm{i}^{\text {th }}$ column in $\mathrm{A}^{\mathrm{T}}$.

\subsection{Flipping of an image}

The image of road signs and number plates appear the wrong way when viewed from the rear-view mirror of a vehicle. Also, when you look at a text in a mirror, a flipped image or reverse image, is seen. For example, as in Figure 1, n the ambulance van, the mirror image of the word AMBULANCE is displayed, so that drivers of the other vehicles can pave way for the van.

\section{ヨЭИА」UЯMА}

Figure 1: Mirror image of the word AMBULANCE

\subsection{Python programming language}

Python is an object-oriented programming language created by Guido van Rossum which is known for its simplicity. It can be learnt easily by a layman. It is a high-level language used for web and app development. Python code has the feature of getting translated into other programming languages easily.It can also be used for various applications involving the display of images, processing text, solving equations and for saving enormous data.

Additionally, Python supports the use of modules and packages which can be easily installed. In other words, 
programs can be designed, and code can be reused across a variety of projects. It is widely used as it is simple and easy to use.

\section{MATRIX OPERATION}

Given any matrix, reverse each row of the matrix. For example, the row $[1,2,3]$ results in $[3,2,1]$ by reversal. This results in the flipping of the image horizontally.

To get a horizontal flip, do the matrix rotation by $90^{\circ}$ in the anti-clockwise direction and then take its transpose.

Step 1: Matrix rotation by $90^{\circ}$ in the anti-clockwise direction

Consider the matrix $A=\left[\begin{array}{lll}1 & 2 & 3 \\ 4 & 5 & 6 \\ 7 & 8 & 9\end{array}\right]$.

Take the transpose of matrix A and call it B.

$$
B=A^{T}=\left[\begin{array}{lll}
1 & 4 & 7 \\
2 & 5 & 8 \\
3 & 6 & 9
\end{array}\right]
$$

Find the horizontal reflection of matrix B and call it as C.

\section{Step 2:}

$$
C=\left[\begin{array}{lll}
3 & 6 & 9 \\
2 & 5 & 8 \\
1 & 4 & 7
\end{array}\right]
$$

Taking the transpose of the matrix $\mathrm{C}$ results in the desired outcome:

$$
C^{T}=\left[\begin{array}{lll}
3 & 2 & 1 \\
6 & 5 & 4 \\
9 & 8 & 7
\end{array}\right]
$$

\section{PYTHON PROGRAM}

A python code is created which transforms a mirrored image into a corrected original image. For example, Figure 2 shows the mirrored image of the binary tree and its corrected image got by flipping the image.

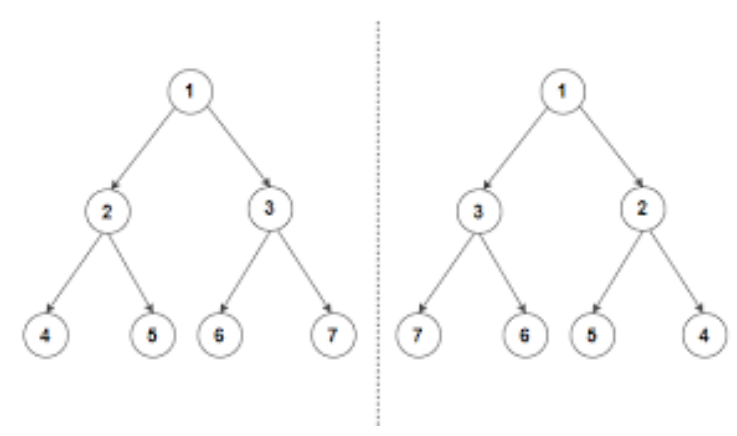

Figure 2: A binary tree and its flipped image
Originally, every image can be represented as a 3D array of binary values from 0 to 255 . The computer image is digitalized by converting the color picture into a three $\mathrm{m}$ by $\mathrm{n}$ matrices of red, green and blue components [6]. This decomposed matrix when subject to matrix operation results in the flipping of images.

The matrix operation used in this study are row operation and transpose of a matrix. These two matrix operations are being applied per RGB component.

Initially, save the photo, which is a mirrored image, in a directory. The following Python Program opens the mirrored picture, flips it horizontally and finally saves a corrected copy in the same directory.

The picture is saved as scenary.png. As soon as the program is run, the file named corrected.png is created. When this file is opened, we could notice that it is the correct image got by flipping the mirror image.

Figure 3 shows the Python code used to flip an image.

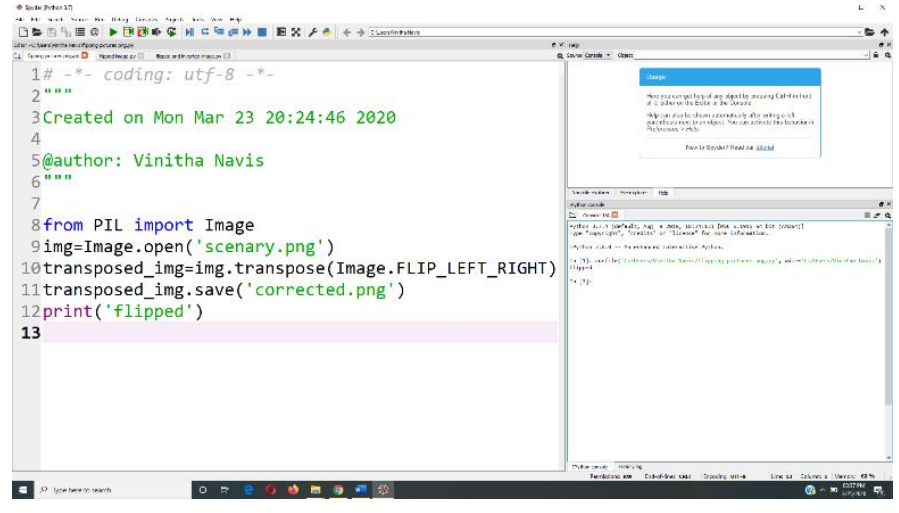

Figure 3: Python code

The outcome of the code is given below:

Figure 4 shows the mirrored image of the scenery and its corrected image got by flipping the image using the above python code. 

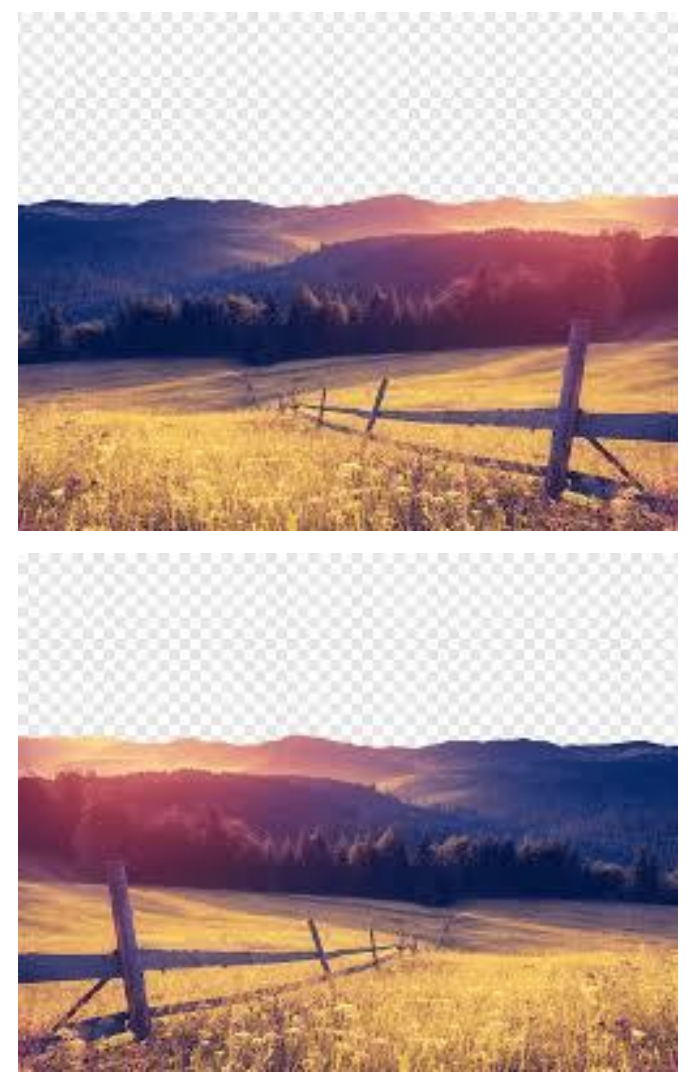

Figure 4: Mirrored image and the corrected image of the scenery.

\section{APPLICATION}

The above concept can be performed by many apps which can be downloaded from the play store. However, there is a possibility for others to misuse our photo. This can be avoided by correcting the image using the python programming language which can be downloaded at home.

\section{CONCLUSION}

Using the Python Programming Language, the flipping of an image can be done with the help of row operation and taking transpose of a matrix.

\section{REFERENCES}

1. R. Jothikumar, Siva Shanmugam. G, M.Nagarajan, S.Premkumar, A.Asokan, Analyzes of Mouth Cancer Using Max-Min Composition in Soft Computing, International Journal of Advanced Trends in Computer Science and Engineering, Volume 8, No.3, May - June 2019.

https://doi.org/10.30534/ijatcse/2019/76832019

2. Sukhada Chokkadi, Sannidhan MS, Sudeepa K B, Abhir Bhandary, A Study on various state of the art of the Art Face Recognition Systemusing Deep Learning Techniques,
International Journal of Advanced Trends in Computer Science and Engineering, Volume 8, No.4, July - August 2019.

https://doi.org/10.30534/ijatcse/2019/84842019

3. Sanil Shanker KP, An Algorithm to Transpose Zero- One Matrix, International Journal of Computer Science and Information Technologies, Vol. 7(4), 2016, 19601961

4. Transpose - from Wikipedia, the free encyclopedia.

5. Ahmed Sherif Zekri, Enhancing the Matrix transpose operation Using INTEL AVX Instuction Set Extension, International Journal of Computer Science and Information Security, Volume 6, Issue 3(2014). https://doi.org/10.5121/ijcsit.2014.6305

6. Ravimal Bandara, Lochandaka Ranathunga, Nor Aniza Abdullah, Nature Inspired Dimensional Reduction Technique for Fast and Invariant Visual Feature Extraction, International Journal of Advanced Trends in Computer Science and Engineering, Volume 8, No.3, May June 2019

https://doi.org/10.30534/ijatcse/2019/57832019 